\title{
Spatial SPION Localization in Liposome Membranes
}

\author{
Cécile Bonnaud $^{1,2}$, Dimitri Vanhecke ${ }^{1}$, Davide Demurtas ${ }^{3}$, Barbara Rothen-Rutishauser ${ }^{1,4}$, and Alke Petri-Fink ${ }^{1,2}$ \\ ${ }^{1}$ Adolphe Merkle Institute, University of Fribourg, Marly 1723, Switzerland \\ ${ }^{2}$ Chemistry Department, University of Fribourg, Fribourg 1700, Switzerland \\ ${ }^{3}$ Interdisciplinary Center for Electron Microscopy, École Polytechnique Fédérale de Lausanne, Lausanne 1015, Switzerland \\ ${ }^{4}$ Respiratory Medicine, Bern University Hospital, Bern 3010, Switzerland
}

\begin{abstract}
Nanocarriers, including liposomes, offer great opportunities for targeted and controlled therapy. The development in this field has led to a large panel of drug delivery systems, which can be classified into 3 different nanovector generations. However, the success of such smart materials requires the control of a large variety of properties and parameters. Unfortunately, characterization at the nanoscale is often cumbersome and many methods are still being developed. Liposomes have been characterized by cryogenic electron microscopy (CryoTEM) for quite some time, also in combination with nanoparticles, in particular with superparamagnetic iron oxide nanoparticles (SPIONs) incorporated inside the liposomal membrane. CryoTEM, unlike classical TEM, maintains the native state of the liposomes. The quick freezing of the sample immobilizes particles and liposomes exactly at their position in the suspension. Therefore, localization information can be extracted from the images. However, data must be treated extremely carefully keeping in mind that 2-D projections of a 3-D object are observed. In this paper, we discuss the analysis of cryoTEM images of liposome-particle hybrids, including the estimation of the contrast transfer function (CTF) and electron dose, as well as the correct positioning of the sample holder and tomography for accurate localization.
\end{abstract}

\section{INTRODUCTION}

$\mathbf{L}$ IPOSOMES are drug carriers which are mostly used to circumvent problems arising from drug solubility and degradation [1]. As a consequence, liposomal drug formulations are increasingly active on the market as carriers for drug delivery [2]. The advantages of these vesicles are myriad: they self-assemble from phospholipids, are biocompatible and have the ability to accumulate in selected tissues, for example tumors, resulting in therapeutic index enhancement of anticancer drug [3], [4]. The drug concentration at the target is increased and exposure of normal host tissues is decreased, reducing drug systemic toxicity [2]. Despite important breakthroughs in the field, many different aspects such as circulation time, targeting efficiency/accuracy or controlling drug release are still heavily investigated both in academia and industrial research and development. To produce long-circulating carriers, liposomes with modified surfaces have been developed. In particular, surface grafted polyethyleneglycol (PEG) has been shown to extend blood-circulation time while reducing mononuclear phagocyte system uptake [5], [6]. The current generation of long-circulating liposomes only passively targets the incorporated molecules to the site of action by extravasation in tumors, such as e.g. Doxil for metastatic ovarian cancer [4]. So-called active targeting of liposome-encapsulated drugs is generally accomplished by coupling targeting agents, such as antibodies or other ligands to vesicles [4], [7]. In particular the control of cargo release is paramount since the load must remain inside the liposomes until the target is reached [8]. Uncontrolled release in the bloodstream leads to fast elimination and capture by the cells of the immune system and significantly reduces efficiency. Ideally, signaling originating directly from the liposome triggers the release. First and second-generation liposome nanovectors are passively triggered in response to the tumor environment, such as lower $\mathrm{pH}$ [9] or higher temperature [6], [10]. Third generation liposome nanovectors promise to be responsive e.g. to superparamagnetic iron oxide nanoparticles (SPIONs) [11], excited by a noninvasive external stimulus such as e.g. a magnetic field [12]. When such magnetic nanoparticles are subjected to a magnetic alternating current (ac) field they can show remarkable heating effects related to losses during the magnetization reversal process of the particles [13], [14]. So-called magnetoliposomes [15] i.e. thermo-sensitive vesicles composed of a phospholipid membrane encapsulating magnetic nanoparticles, become permeable upon exposure to an ac-field [16], [17]. Relaxation processes of the magnetic nanoparticles result in heat production in the vicinity of the liposome membrane, which destabilizes and eventually releases the encapsulated drug. However, the concentration of SPIONs required to open the liposomal membrane is very high and particles may even impair the co-encapsulated drug prior to their release, for instance in the case of thermolabile drugs. One approach to optimize this magnetically triggered release consists in immobilizing ultrasmall magnetic nanoparticles between the bilayers of the liposome membrane [12], [18]. Heat is locally created only inside the membrane thus ensuring an improved protection of the cargo. Additionally, SPIONs are widely used as contrast agents in magnetic resonance imaging (MRI) and can therefore be a tool for image guided drug delivery [19]-[21]. However, precise characterization of these highly complex constructs is not straightforward. Von White $\mathrm{G}$ 2nd et al. just recently demonstrated the impact of nanoparticles encapsulated in the liposomal membrane on its transition phase temperature $(\mathrm{Tm})$ [22]. Standard characterization techniques, 
such as dynamic light scattering (DLS), small angle X-ray and neutron scattering (SAXS and SANS) render statistical relevant information about the suspension [23] but fail to provide in situ information about localization ("how and where are the magnetic nanoparticles localized in a single liposome?") as well as quantification ("how many particles can be immobilized per liposome?").

So far, classic transmission electron microscopy (TEM) with chemically fixed and dehydtrated samples has been used to visualize magnetic particles inside liposomes [24], however, this method is of limited value. Especially the reaction of chemical fixatives with lipids is weak. Common fixatives such as glutaraldehyde and formaldehyde bind poorly to lipids [25], and if at all, unsaturated lipids are preferentially bound [26], [27]. With poor fixation of both water and lipids, severe extraction of the only two constituents of liposomes occurs during the subsequent dehydration step [28]. Cryo-transmission electron microscopy (cryoTEM) is a more suitable approach, since the dehydration step is avoided and its accompanied lipid extraction effect: it allows for the observation of hydrated samples [29]. Furthermore, liposomes are sufficiently small to allow plunge freezing [30] and do not require any sectioning process. However, the increased complexity of the third generation liposome nanovectors, especially the precise co-localization of small (nano)particles into a bilayer, demands for an increased accuracy of the in situ information. In this work, we describe a method to improve the spatial visualization of liposome-SPIONs hybrids by cryoTEM and cryo electron tomography (cryoET) providing nanometer precise qualitative and quantitative information at the local scale. This is a prerequisite for the assessment and quality control of the third generation liposome nanovectors.

\section{MATERIAL AND MEthods}

1) Oleic Acid Coated SPIONs Synthesis (OA-SPIONs): SPIONs were synthesized by thermal decomposition of the metal-oleate precursor in a high boiling solvent following the procedure of Park et al. [31]. In a typical synthesis of iron-oleate complex, $2.165 \mathrm{~g}$ of $\mathrm{FeCl} 3.6 \mathrm{H} 2 \mathrm{O}$ (Sigma Aldrich,98\%) and $7.443 \mathrm{~g}$ of sodium oleate (TCI,97\%) was dissolved in a solvent mixture (ethanol/Milli-Q water/hexane $=16 \mathrm{~mL} / 12 \mathrm{~mL} / 28 \mathrm{~mL}$ ) and heated to $70^{\circ} \mathrm{C}$ for $4 \mathrm{~h}$. The organic phase containing the complex was washed 3 times with $10 \mathrm{~mL}$ of Milli-Q water, hexane was removed and replaced by $52.6 \mathrm{~mL}$ octyl ether $\left(287^{\circ} \mathrm{C}\right) .1 .4$ $\mathrm{ml}(4.39 \mathrm{mmol})$ oleic acid (Sigma Aldrich 90\%) was added to yield a complex/oleic acid ratio of $2: 1$. The mixture was gradually heated $\left(30-135^{\circ} \mathrm{C}, 9.00^{\circ} \mathrm{C} / \mathrm{min}\right.$ and $135^{\circ} \mathrm{C}-287^{\circ} \mathrm{C}$, $2.95^{\circ} \mathrm{C} / \mathrm{min}$ ) to $287^{\circ} \mathrm{C}$ and kept at this temperature for 30 minutes. The resulting particle containing suspension was cooled to room temperature and subsequently purified by precipitation-resuspension cycles. Particles with a core size of $5.7+/-1.0 \mathrm{~nm}$ were dispersed in chloroform to a final concentration of $2.5 \mathrm{mg}$ iron $/ \mathrm{mL}$.

2) Liposomes Synthesis: Thin Layer Evaporation (TLE): Liposomes were prepared by a conventional thin lipid film rehydration method [32]. Briefly, cholesterol (Sigma Aldrich,
99\%) $10 \% \mathrm{w}(5.2 \mu \mathrm{mol}), 1,2$-dipalmitoyl-sn-glycero-3-phosphocholine or DPPC (Aventi polar lipids) $43 \% \mathrm{w}$ (12.3 $\mu \mathrm{mol})$, 1,2-dipalmitoyl-sn-glycero-3-phospho-(1'-rac-glycerol) or DPPG (Aventi Polar lipids) 24\%w (7.3 $\mu \mathrm{mol})$, 1,2-dimyristoyl-sn-glycero-3-phosphoethanolamine or DMPE 19\%w $(6.3 \mu \mathrm{mol})$, 1,2-dipalmitoyl-sn-glycero-3-phosphoethanolamine-PEG-2000 or DPPE-PEG-2000 (aventi polar lipids) $5 \% \mathrm{w}(0.4 \mu \mathrm{mol})$ were mixed in chloroform. The solvent was evaporated in a rotary evaporator $\left(50^{\circ} \mathrm{C}, 500 \mathrm{mbar}, 24 \mathrm{~h}\right)$ to form a lipid film. A mixture of Tris-Acetate (Sigma-Aldrich, reagent grade) and sodium chloride (Sigma-Aldrich, 99\%) with concentrations of $40 \mathrm{mM}$ and $100 \mathrm{mM}$, respectively was added to the lipid film at $55^{\circ} \mathrm{C}$ to obtain a lipid concentration of $10.5 \mathrm{mg}$ lipids $/ \mathrm{mL}(15.8 \mu \mathrm{mol} / \mathrm{mL})$. Rehydration required vigorous shaking at $55^{\circ} \mathrm{C}$ until all lipids were suspended as multi-lamellar vesicles (MLV). To obtain $100 \mathrm{~nm}$ unilamellar vesicles (LUV), a mini-extruder (aventi polar lipids) with polycarbonate membranes of different pore sizes $(0.8 \mu \mathrm{m}, 0.4$ $\mu \mathrm{m}, 0.2 \mu \mathrm{m}, 0.1 \mu \mathrm{m})$ was used. After equilibration at $55^{\circ} \mathrm{C}$, 15 passes through each membrane were performed to ensure a narrow size distribution. Liposomes were stored at $4^{\circ} \mathrm{C}$ and were stable for more than 5 weeks. The bilayer thickness is 5.7 $\pm 0.2 \mathrm{~nm}$.

3) Particles Incorporation Into Membranes: Two different strategies were followed to investigate the incorporation efficiency of ultrasmall hydrophobic particles into liposome bilayers. The first method was proposed by Bibi et al. who dried lipids together with hydrophobic particles, followed by ultrasound assisted rehydration with the buffer at $55^{\circ} \mathrm{C}$ [8]. The second method relied on the presence of surfactants and was inspired by membrane protein incorporation [33], [34]. Therefore, OA-SPIONS were first transferred to the buffer with the help of a surfactant by gently evaporating the solvent in contact with the buffer ( $\beta$-octylglucoside, $128 \mathrm{mM},[\mathrm{Fe}]=1.02 \mathrm{mg} / \mathrm{mL})$. Surfactant molecules covered the OA-SPIONs, which allowed the dispersion in the buffer. Possible ligand exchange between the oleic acid and the surfactant could occur but was not changing the final result. In a second step, these particles were mixed with either micelles of phospholipids-surfactant or pre-formed liposomes. The surfactant was then dialyzed to initiate particlephospholipids assembly. In the case of surfactant micelles, liposomes are forming along the process, including particles in the assembly.

4) Cryo-Transmission Electron Microscopy: An EM grid with holey carbon film was held at the rim with tweezers and 4-5 $\mu \mathrm{L}$ of sample solution was applied on the grid. The tweezers were mounted in an automatic plunge freezing apparatus (Vitrobot, FEI, The Netherlands), which controled humidity and temperature. Excess sample was removed by application of a filter paper ("blotting"). After blotting, the grid was immediately immersed in a small metal container filled with liquid ethane, which was cooled from the outside by liquid nitrogen. The speed of cooling is such that crystalline ice does not have the time to form.

CryoTEM images and stereoscopic views were recorded at $-170^{\circ} \mathrm{C}$ in a Philips CM12 EM at $100 \mathrm{kV}$ on an Eagle $4098 \mathrm{X}$ 4098 pixel digital camera (FEI, Eindhoven, The Netherlands). CryoET was performed in a Tecnai F20 (Eindhoven, The 
Netherlands) at $-170^{\circ} \mathrm{C}$ operating at $200 \mathrm{kV}$ equipped with a cryo-specimen holder Gatan 626 (Warrendale, PA). Digital images were recorded with a GatanMultiScan charge coupled device (CCD) camera 1024 X 1024 SPIONs in the tilt series were used as fiducial markers for alignment and reconstruction (weighted back projection) was performed using iMOD [35].

\section{RESULTS AND DISCUSSION}

Fig. 1(b) shows liposomes with SPIONs that were added inside the lipid film, whereas Figs. 2to 5 illustrate the results of the surfactant mediated incorporation strategy, from phospholipid-surfactant micelles (Fig. 2) to pre-formed liposomes (Figs. 3-5). A macroscopic color change can be observed upon adding SPIONS to a liposomal suspension: the initial whitish-clear suspension turns brownish (compare the insets of Fig. 1). It has been argued that this color change results from suspending the hydrophobic particles in the aqueous solvent, thereby proving the interaction between particles and liposomes [18]. However, the color change could also arise from small nanoparticle aggregates $(12-100 \mathrm{~nm})$ dispersed in the suspension after sonication. Such aggregates are commonly observed in cryoTEM [Fig. 1(b)]; they do not have influence sample turbidity or necessarily result in sedimentation. Hence, macroscopic inspection is not sufficient to demonstrate liposome-SPION interaction. Consequently, evidence for the incorporation of particles in membranes must be evaluated by techniques that provide high resolution in situ information, such as transmission electron microscopy. CryoTEM has previously been used as a methodology to determine liposomal nanovector characterization. Chen et al. showed energy-dispersive X-ray spectroscopy (EDX) of single liposomes in cryoTEM [18]. Despite the high spectral information (their data proved that iron was present), the spatial resolution (about $150 \mathrm{~nm}$ ) was insufficient to draw any conclusions about the distance between SPIONs and membrane or incorporation in the bilayer. Amstad et al. investigated liposome-SPION systems using cryoTEM [12]. However, the relatively low magnification as well as the lack of defocus settings and diffractograms make this interpretation ambiguous. Fig. 2 shows the impact of focal settings on membrane contrast. The focal settings of the electromagnetic lenses contribute to an optical effect that is described by the contrast transfer function (CTF). Being a function of the focus settings, the CTF results in changes in the amplitude and phase of the signal. An optical artifact can be created if the object is imaged in overfocus, in which Fresnel fringes appear forming an edge effect seen as a black lining that could be confused as a real object. This could be a source of misinterpretation. Therefore, a focal series was recorded to demonstrate that the black line is a real object and no artifact. On the contrary, a white line is outlining edges in defocus. The width of the lining increases with increasing defocus. The focal series in Fig. 2 shows an increasing width with increasing defocus, indicating that the thin membrane around the SPIONS is a real object. Particular for liposomes, this optical effect could be misinterpreted as a surrounding bilayer. This is illustrated in Fig. 3, where two nanoparticles appear to co-localize with the membrane bilayer. Even at a higher magnification it remains unclear if the SPIONs are indeed between the membrane bilayer sheets or if it can be attributed to the defocus
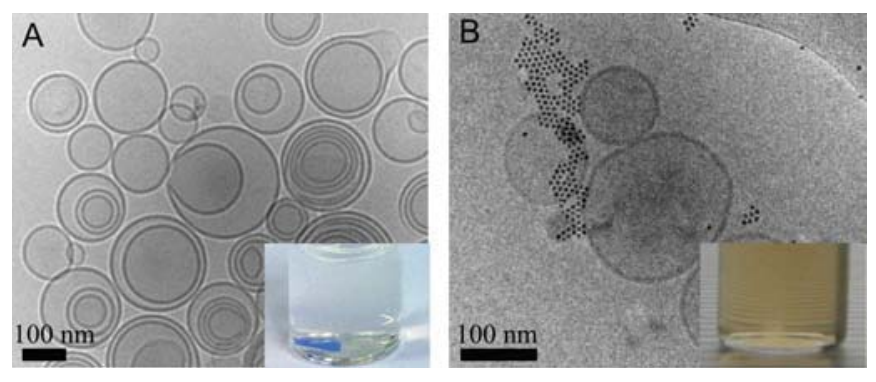

Fig. 1. Cryo-TEM images and corresponding macro-digital pictures of a liposome suspension either without oleic acid SPIONs (a) or after incorporation of oleic acid coated SPIONs (b) using the lipid film-particle rehydration method. The brownish color of the suspension (compare the insets of A and B) is attributed to either SPIONs inside the vesicles or to aggregates of particles in the suspension.

effect. In any case, it is impossible to demonstrate the localization of the SPIONs in 3-dimensional liposomes based on 2-dimensional projections: even without optimal defocus settings, this question cannot be answered with merely one projection.

Attempts to avoid 3-D misinterpretation were provided by Wu et al. who tilted the sample holder to visualize a hollow gold nanoshell on a liposome surface [11]. Such a tilting approach generates a pair of angled images, which are interpreted by the brain as having the perception of depth. In Fig. 4, such a stereoscopic pair was recorded by tilting the sample with angle $(\theta)$ from $-30^{\circ}$ to $+30^{\circ}$ in the microscope. The apparent association between liposome membrane and a nanoparticle [Fig. 4(a)] is excluded by the observation at $30^{\circ}$ tilting angle, where the cluster is now projected outside the liposome [Fig. 4(b)]. The procedure allows for localization of clusters of particles relative to the liposome, as shown in Fig. 4(c), (d), where the tilting pair shows that the membrane and the cluster are co-localizing. Fig. 5 summarizes the geometric details of the stereoscopic tilting effect in a SPION-liposome. This scheme already shows that particles above (or below) liposomes can be projected at the same position than the membrane. Particles, which are not associated with the membrane, demonstrate in the projected image an angle-dependent shift relative to the membrane. If particles are observed outside the liposome at one tilt angle, it can be for sure assumed that they are not incorporated inside the membrane. Particles within the membrane are projected at least at one tilt angle with the membrane, unlike particles inside the liposome. However, if particles remain inside the liposome at chosen tilt angles, we cannot say whether it is encapsulated in the liposome or inside the membrane as a full tilt $\left(+90 /-90^{\circ}\right)$ is not possible. If the position of particles allows getting two images with particles projected at the same position than the membrane, it can be claimed that they are co-localized. Therefore, at least the recording of a stereoscopic pair is required in order to make a statement on the localization of particles relative to a 3-D object such as a liposome. However, the tilt angle must be carefully chosen to allow particle-membrane localization.

The axial resolution can be improved by increasing the number of tilt angles. This approach is known as cryo-electron tomography [36]. High 3-D resolution is necessary for the confirmation of the interaction of a single particle with the liposome membrane [37]. This technique is particularly useful 

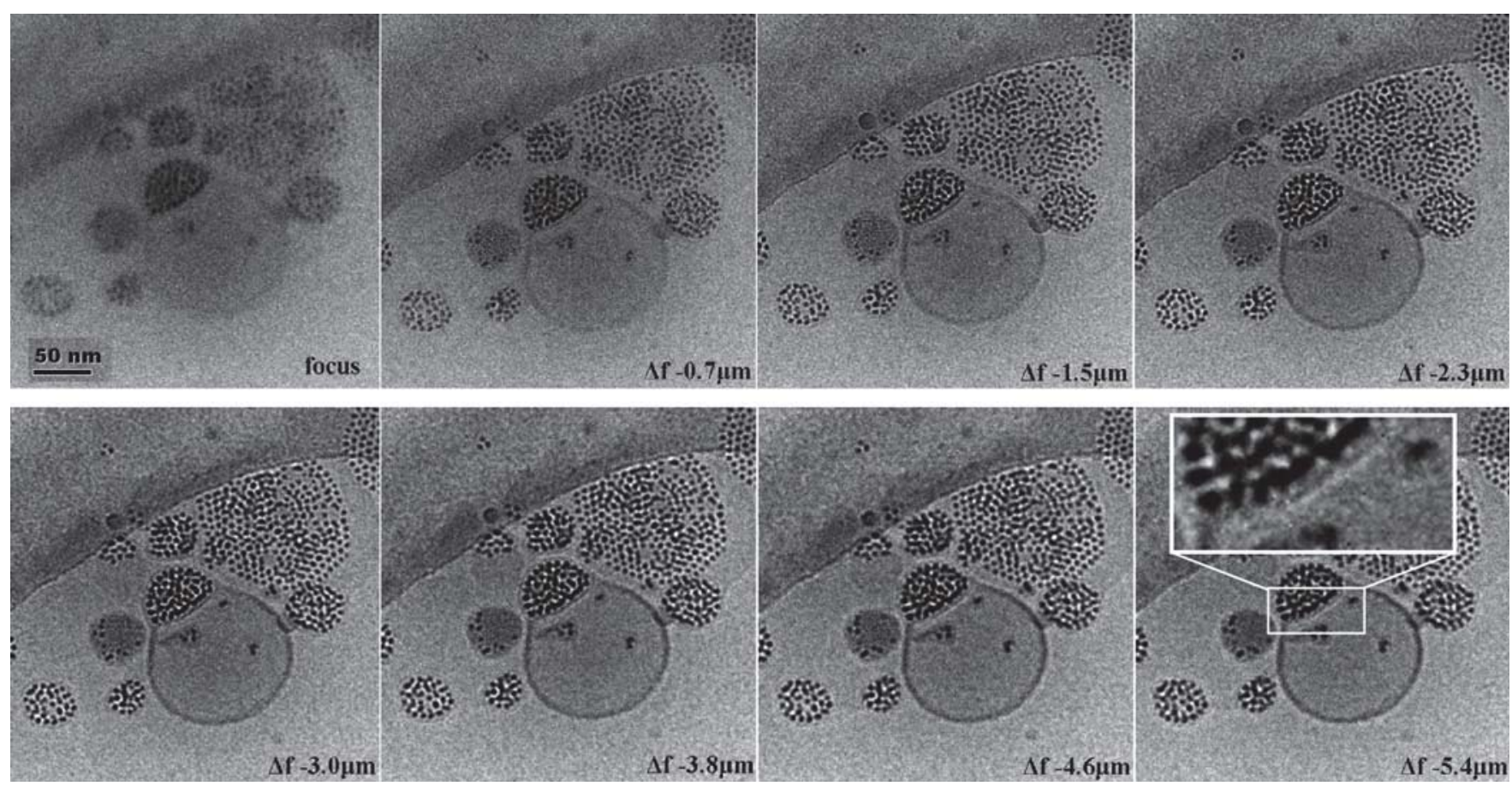

Fig. 2. Cryo-TEM focal series of a liposome with associated SPIONs. The liposome was constructed by dialyzing the surfactant from the phospholipid-surfactantparticles micelles. By increasingly higher defocus settings, the objects in the image become increasingly better delineated (which is experienced as an increase in contrast). At the suitable defocus settings, the two layers of the membrane part and engulf an aggregate of SPIONs (see inset).

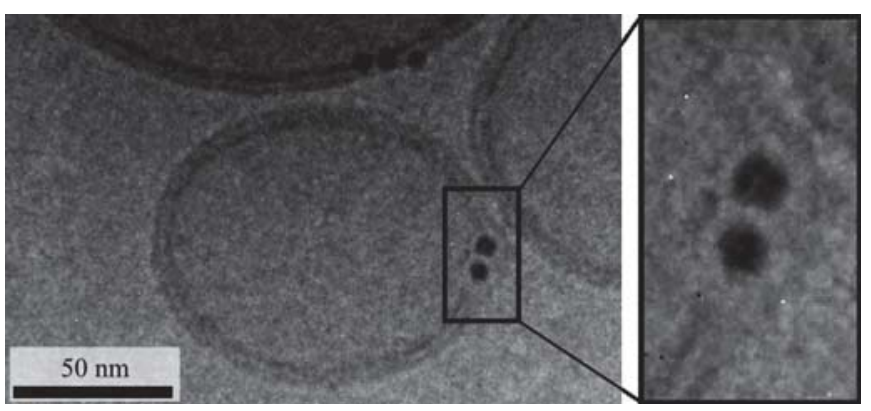

Fig. 3. Cryo-TEM of SPIONs and liposomes (same preparation as Fig. 2) at a defocus of $-2 \mu \mathrm{m}$. Although a putative bilayer appears around the SPIONs, it cannot be excluded that this is an optical effect of the defocus and therefore not a real structure.

in cases where only one or a few particles interact with the membrane. Fig. 6 shows 3 orthogonal digital slices (XY or top view, $\mathrm{XZ}$ or front view, YZ or side view) of a liposome with SPIONs. Again, one top view is not sufficient, but together with the front and side views we can clearly localize a single SPIONs in situ. Therefore, CryoET solves the question of localization ("how and where are the magnetic nanoparticles localized in a single liposome?") as well as their quantification ("how many particles can be immobilized per liposome?"). However, both the powerful CryoTEM and CryoET techniques are limited by sample thickness, water state and electron dose. Samples with a thickness up to a few hundred nanometers are readily observed in modern 200 and $300 \mathrm{kV}$ cryoTEM, but thicker samples are challenging and even high-end, state-of-the art instrumentation cannot routinely handle samples thicker than about $1 \mu \mathrm{m}$. Then again, liposomes rarely exceed $200 \mathrm{~nm}$ in thickness and hence are well suited for cryoTEM and cryoET. In addition,
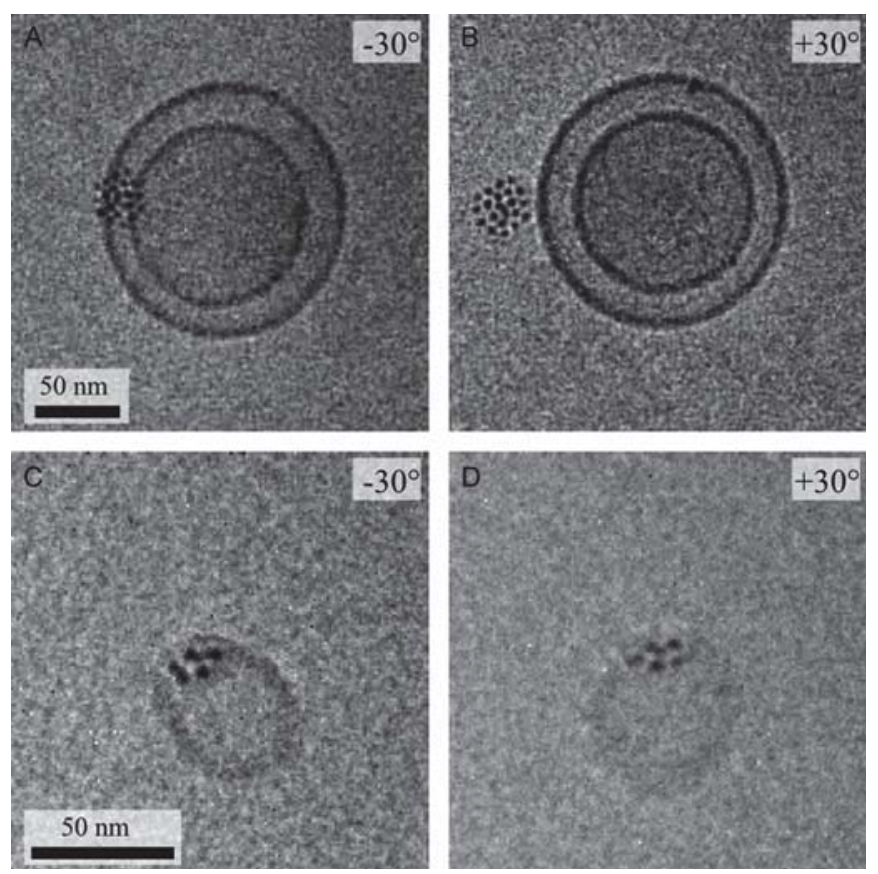

Fig. 4. Cryo-TEM images of SPIONs and liposomes (prepared as Fig. 2) at tilt angles of $-30^{\circ}$ (a, c) and $+30^{\circ}$ (b, d). (a) Although particles at $-30^{\circ}$ seem to be associated with the liposome membrane, the tilt image at $30^{\circ}$ (b) challenges this interpretation: it is the loss of the third dimension during projection which leads to this misinterpretation. (c) Again, a cluster of particles seemingly interacts with the liposome membrane in the $-30^{\circ}$ tilt image. This interpretation is maintained, independent of the tilt angle (d).

good fixation of water is central since it constitutes the main component of the sample. Water in cryofixed samples must be in an amorphous state (in contrast to crystalline ice), which is 


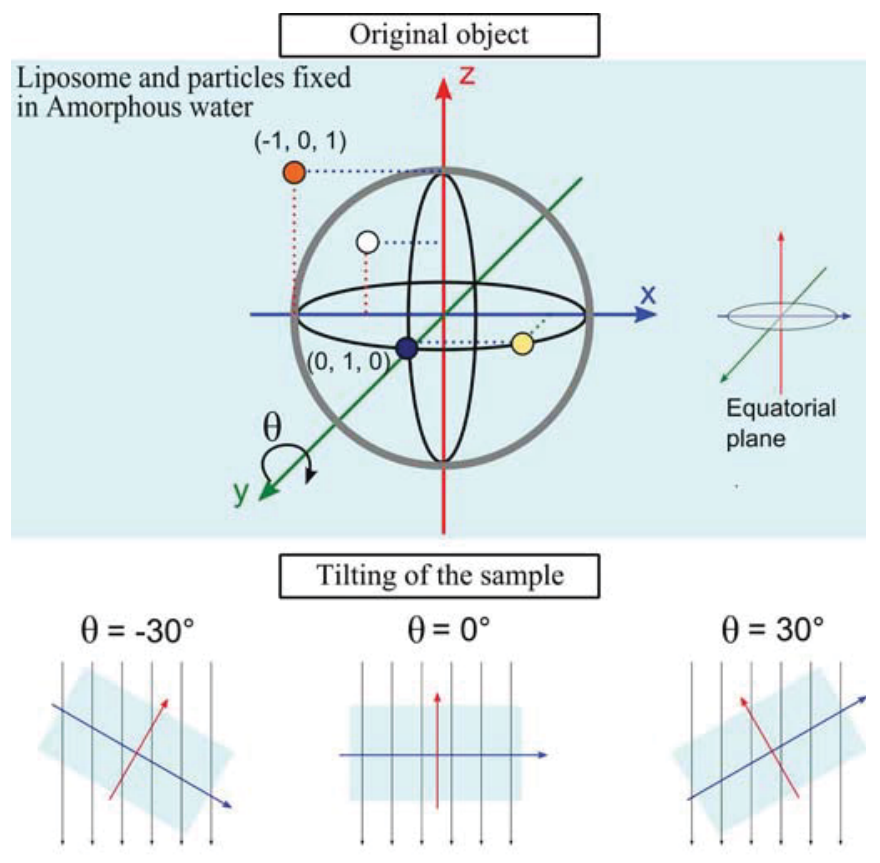

Projected image (top view)
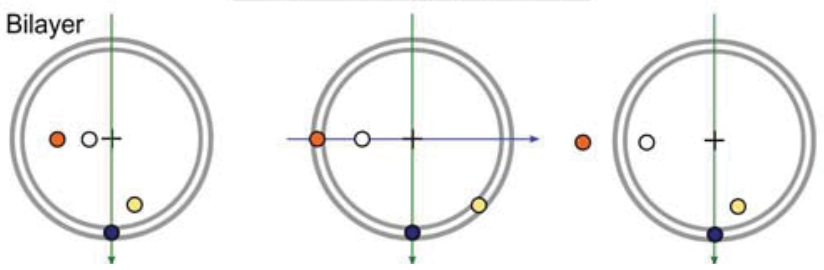

Fig. 5. Schematic representation of the combined tilting-projection effect. On top is a schematic liposome, represented as a sphere, surrounded by several particles: above the liposome (red), inside the liposome (white), inside the membrane (black and yellow). At $0^{\circ}$ projection, with the exception of the white particle, all particles are seemingly associated with the membrane (schematic bilayer representation). This interpretation is true for black and yellow, but not for the red particle. If the sample is tilted, particles will rotate in function of their position and a tilt-dependent shift on the projected image is observed. If particles are above or below the liposome, they will appear outside the liposome at certain tilt angles. A particle inside will never be projected at the same position as the membrane.

usually achieved by cryofixation procedures but should nevertheless be ensured by an electron diffractogram of the sample. Crystalline ice induces morphological changes, which cause for example abrupt angles to membranes. Finally, cryo-fixed samples can only bear a limited electron dose, usually around 50 to 100 electrons per square Angstrom. Higher electron doses evoke immediate but localized destruction of the sample (so-called bubbling). Therefore, meta-data on approximate sample thickness, water state and applied total electron dose should accompany cryo electron micrographs.

\section{CONCLUSION}

Transmission electron microscopy remains crucial for the analysis of liposomal systems and the interaction with colloidal nanoparticles. Delivering sufficient resolution, the data however comes as projections, thereby losing information on the third dimension. This is an obvious but central point for the localization of SPIONs inside membranes. Furthermore, water-based systems such as liposomal solution demand cryo-electron
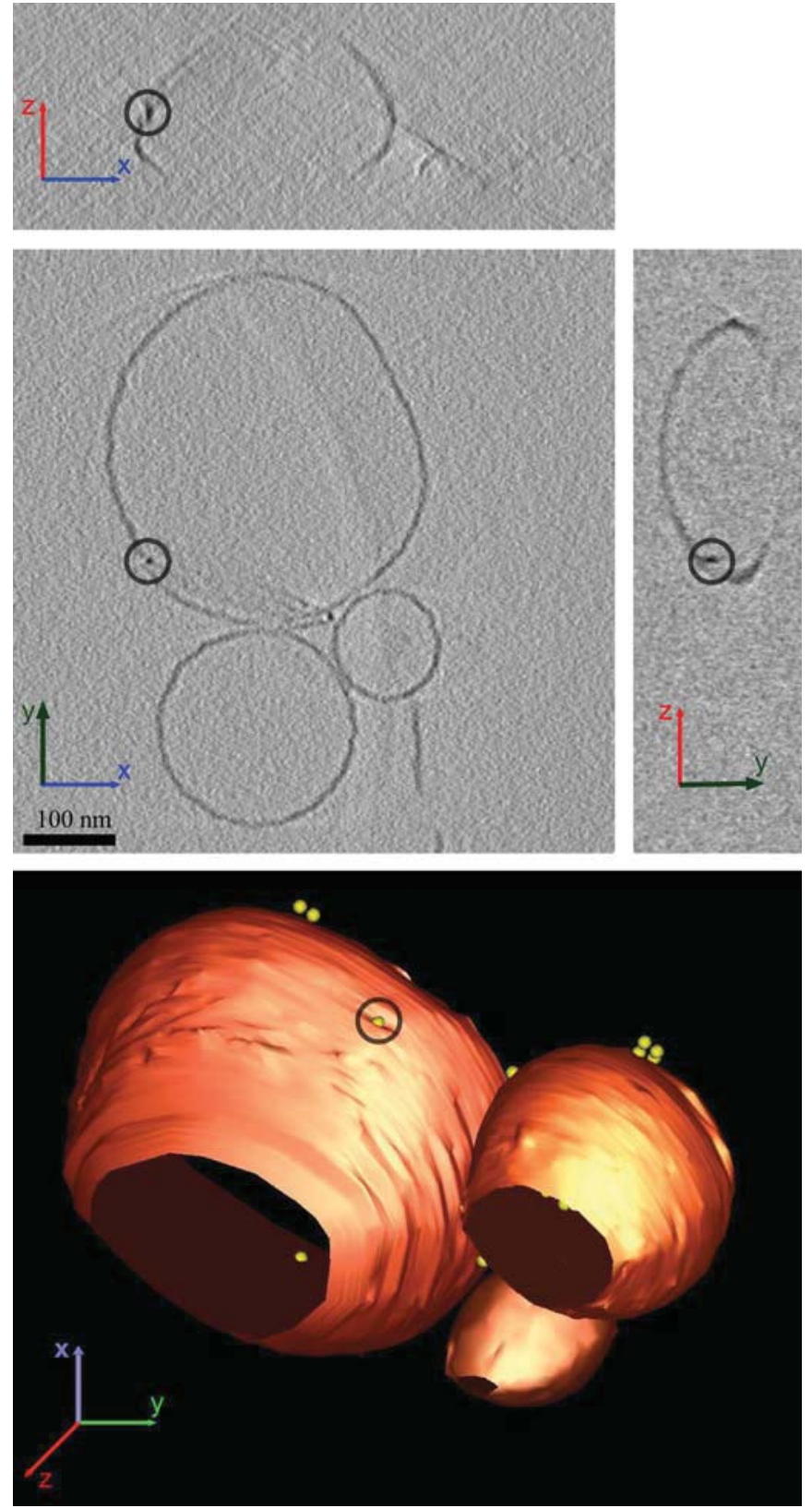

Fig. 6. Cryo-electron tomography and 3-D reconstruction of SPIONs and liposomes (prepared as Fig. 2). The sample holder was tilted between $-60^{\circ} /+60^{\circ}$, with a $3^{\circ}$ increment. The defocus was set at $-3 \mu \mathrm{m}$; the total electron dose was $<100$ e $-/ \AA$. Top: top view (YX), side view (XZ) and front view (YZ) slices from the 3 dimensional stacks. The dark contrast of a SPION in the membrane is seen in all the slices. Below: the rendered model. Polar regions of liposomes are missing due to incomplete sampling during tilt series acquisition $\left(-60^{\circ} /+60^{\circ}\right)$. The model shows that SPIONs (yellow) co-localize in 3-D space with the liposome membrane (red).

microscopy in order to preserve their ultrastructure as close as possible to the native state. Additionally, proper interpretation of the applied defocus is necessary. Phase contrast gives an edge-detecting effect that provides contrast for liposomes but can be mistakenly understood as a membrane bilayer on other objects.

Finally, the electron dose limits the signal to noise ratio, and a noisy image might result in misinterpretation. Tilt stereopairs is the easiest form of a reconstruction of the third dimension and 
is often sufficient. Tilts of $30^{\circ}$ were already enough to prove the interaction or not of particles with membranes. In case where very precise localization (resolution of a few nanometers) is required, the third dimension reconstruction by cryo-electron tomography is highly recommended.

\section{ACKNOWLEDGMENT}

The authors would like to thank the Dr. A. Bretscher Fond and the microscopy imaging center, University of Bern, for the use of the Tecnai F20 cryo-TEM. This work was supported by the Swiss National Science Foundation under Grant NRP 62 (126104) and Grant PP00P2-123373/1, the Adolphe Merkle Foundation, and the University of Fribourg and FriMat (Fribourg Center for Nanomaterials). The authors would also like to thank Prof. H. Vogel and Dr. R. Hovius (Laboratory of Physical Chemistry of Polymers and Membranes, Ecole Polytechnique Fédérale de Lausanne, EPFL, Switzerland) for their generous supply of phsopholipids and their support with the liposomes.

\section{REFERENCES}

[1] T. M. Allen, "Liposomal drug formulations. Rationale for development and what we can expect for the future," Drugs, vol. 56, no. 5, pp. 747-756, 1998

[2] H. I. Chang and M. K. Yeh, "Clinical development of liposome-based drugs: Formulation, characterization, and therapeutic efficacy," Int. J. Nanomedicine, vol. 7, pp. 49-60, 2012.

[3] N. Desai, "Challenges in development of nanoparticle-based therapeutics," Aaps J., vol. 14, no. 2, pp. 282-295, 2012.

[4] D. Peer et al., "Nanocarriers as an emerging platform for cancer therapy," Nature Nanotechnol., vol. 2, no. 12, pp. 751-760, 2007.

[5] M. L. Immordino, F. Dosio, and L. Cattel, "Stealth liposomes: Review of the basic science, rationale, and clinical applications, existing and potential," Int. J. Nanomedicine, vol. 1, no. 3, pp. 297-315, 2006.

[6] V. P. Torchilin, "Recent advances with liposomes as pharmaceutical carriers," Nature Reviews Drug Discovery, vol. 4, no. 2, pp. 145-160, 2005.

[7] A. Sharma and U. S. Sharma, "Liposomes in drug delivery: Progress and limitations," International Journal of Pharmaceutics, vol. 154, no. 2, pp. 123-140, 1997.

[8] S. Bibi et al., "Trigger release liposome systems: Local and remote controlled delivery?," J. Microencapsulation, vol. 29, no. 3, pp. 262-276, 2012.

[9] D. X. Liu and L. Huang, "Role of cholesterol in the stability of Ph-sensitive, large unilamellar liposomes prepared by the detergent-dialysis method," Biochimica Et Biophysica Acta, vol. 981, no. 2, pp. 254-260, 1989.

[10] G. A. Koning et al., "Hyperthermia and thermosensitive liposomes for improved delivery of chemotherapeutic drugs to solid tumors," Pharmaceutical Research, vol. 27, no. 8, pp. 1750-1754, 2010.

[11] G. Wu et al., "Remotely triggered liposome release by near-infrared light absorption via hollow gold nanoshells," J. Am. Chem. Soc., vol. 130, no. 26, pp. 8175-8177, 2008.

[12] E. Amstad et al., "Triggered release from liposomes through magnetic actuation of iron oxide nanoparticle containing membranes," Nano. Lett., vol. 11, no. 4, pp. 1664-1670, 2011.

[13] R. Hergt et al., "Magnetic particle hyperthermia: Nanoparticle magnetism and materials development for cancer therapy," J. Physics Condensed Mat., vol. 18, no. 38, pp. S2919-S2934, 2006.

[14] S. Laurent et al., "Magnetic fluid hyperthermia: Focus on superparamagnetic iron oxide nanoparticles," Advances in Colloid and Interface Science, vol. 166, no. 1-2, pp. 8-23, 2011.
[15] A. Wijaya and K. Flamad-Schifferli, "High-density encapsulation of Fe3O4 nanoparticles in lipid vesicles," Langmuir, vol. 23, no. 19, pp. 9546-9550, 2007.

[16] L. A. Tai et al., "Thermosensitive liposomes entrapping iron oxide nanoparticles for controllable drug release," Nanotechnology, vol. 20, no. 13,2009

[17] S. Nappini et al., "Magnetoliposomes for controlled drug release in the presence of low-frequency magnetic field," Soft Matter, vol. 6, no. 1, pp. 154-162, 2010

[18] Y. J. Chen, A. Bose, and G. D. Bothun, "Controlled release from bilayer-decorated magnetoliposomes via electromagnetic heating," Acs, Nano,, vol. 4, no. 6, pp. 3215-3221, 2010.

[19] S. J. Soenen et al., "Magnetoliposomes as magnetic resonance imaging contrast agents," Wiley Interdisciplinary Reviews-Nanomedicine and Nanobiotechnology, vol. 3, no. 2, pp. 197-211, 2011.

[20] X. A. Xie and C. F. Zhang, "Controllable assembly of hydrophobic superparamagnetic iron oxide nanoparticle with mPEG-PLA copolymer and its effect on MR transverse relaxation rate," J. Nanomaterials, 2011.

[21] G. Mikhaylov et al., "Ferri-liposomes as an MRI-visible drug-delivery system for targeting tumours and their microenvironment," Nature Nanotechnol., vol. 6, no. 9, pp. 594-602, 2011.

[22] G. Von White, Y. Chen, J. Roder-Hanna, and G. D. Bothun, "Kitchens, structural and thermal analysis of lipid vesicles encapsulating hydrophobic gold nanoparticles," ACS Nano, vol. 6, no. 6, pp. 4678-4685, Jun. 26, 2012.

[23] S. Nappini et al., "Structure and permeability of magnetoliposomes loaded with hydrophobic magnetic nanoparticles in the presence of a low frequency magnetic field," Soft Matter, vol. 7, no. 10, pp. 4801-4811, 2011.

[24] A. Floris et al., "SPION@liposomes hybrid nanoarchitectures with high density SPION association," Soft Matter, vol. 7, no. 13, pp. 6239-6247, 2011.

[25] M. A. Hayat, Principles and Techniques of Electron Microscopy: Biological Applications. Cambridge: Cambridge Univ. Press, 2000, pp. 28-34.

[26] D. Ones, "The reaction of formaldehyde with unsaturated fatty acids during histological fixation," Histochem J, vol. 1, no. 5, pp. 459-491, 1969.

[27] D. L. White et al., "The chemical nature of osmium tetroxide fixation and staining of membranes by x-ray photoelectron spectroscopy," Biochim Biophys Acta, vol. 436, no. 3, pp. 577-592, 1976.

[28] J. L. A. N. Murk et al., "Influence of aldehyde fixation on the morphology of endosomes and lysosomes: Quantitative analysis and electron tomography," J. Microscopy-Oxford, vol. 212, pp. 81-90, 2003.

[29] J. Dubochet et al., "Cryo-electron microscopy of vitrified specimens," Ouarterly Reviews of Biophys., vol. 21, no. 2, pp. 129-228, 1988.

[30] M. Adrian et al., "Cryo-electron microscopy of viruses," Nature, vol. 308, no. 5954, pp. 32-36, 1984.

[31] J. Park et al., "Ultra-large-scale syntheses of monodisperse nanocrystals," Nature Materials, vol. 3, no. 12, pp. 891-895, 2004.

[32] , D. D. Lasic, Ed., Liposomes-From Physic to Application. New York: Elsevier, 1993.

[33] J. L. Rigaud, M. T. Paternostre, and A. Bluzat, "Mechanisms of membrane-protein insertion into liposomes during reconstitution procedures involving the use of detergents. 2. Incorporation of the light-driven proton pump bacteriorhodopsin," Biochemistry, vol. 27, no. 8, pp. 2677-2688, 1988.

[34] J. Knol, K. Sjollema, and B. Poolman, "Detergent-mediated reconstitution of membrane proteins," Biochemistry, vol. 37, no. 46, pp. 16410-16415, 1998.

[35] J. R. Kremer, D. N. Mastronarde, and J. R. McIntosh, “Computer visualization of three-dimensional image data using IMOD," J. Structural Biology, vol. 116, no. 1, pp. 71-76, 1996.

[36] D. Vanhecke et al., "Cryo-electron tomography: Methodology, developments and biological applications," J. Microsc., vol. 242, no. 3, pp. 221-227, 2011.

[37] O. Le Bihan et al., "Cryo-electron tomography of nanoparticle transmigration into liposome," J. Structural Biology, vol. 168, no. 3, pp. 419-425, 2009. 